\title{
A Taxonomy of Risk in Large UK Industrial Firms
}

\author{
Duarte Trigueiros \\ INESC \\ R. Alves Redol 9-2, 1000 Lisbon, Portugal \\ Phone: +351 (1) 79350 00, Fax: +351 (1) 79647 10, \\ E-Mail:dmt@sara.inesc.pt
}

\begin{abstract}
In this study we breakdown the overall movements of the capital market into four orthogonal forces. Then we use a Multilayer Perceptron (MLP) to explain the sensitivities of firms to each of these forces in terms of accounting information. This research found a strong relationship between specific features of accounting reports of firms and their appraisal by the market. Using size, industrial group, work force and payment patterns as predictors, the MLP explained the sensitivities of firm's returns to these overall forces. However, the map relating features of firms to sensitivities is a complex one. This explains why previous studies, based on linear tools, didn't find such strong relationships. The method developed here opens up the possibility of building a taxonomy of risk.
\end{abstract}

\section{Introduction}

This study relates the co-variance structure of returns on assets traded in capital markets, to some characteristics of those assets. Co-variance is a basic feature of returns. This section introduces the concepts leading to expecting such a relation.

\subsection{Capital Markets and Investment}

Decisions by firms and investors are typically carried out under conditions of uncertainty. Uncertain events whose frequency distribution is known are usually referred to as risky events. The return on assets traded in capital markets is risky since the only information available to investors about future returns is their frequency distribution.

Risk can be measured in several ways. It could be assessed as the variability of future earnings, but the measures of variability with practical use are dependent on the validity of assumptions about the distri- bution of the data. Unfortunately, the distribution of returns on assets is usually leptokurtic, thus allowing the parameterization of central trends but not the one of variability. Risk can also be seen as the sensitivity of an asset's expected return to unanticipated changes in economic forces. This definition is free from assumptions about the distribution of returns. Instead, it implies that there is a model relating risk to the expected return on an asset. In capital markets this is the case.

Investors's perception of risk and the expected return on assets traded in capital markets must be linearly related. Otherwise, arbitrage opportunities would emerge. Roll and Ross (1984) [9], offer a simple explanation for this mechanism. If the expected returns are influenced by more than one economic force, the expected return on each asset must be a linear combination of its sensitivities to such forces. The model relating risk to return based on these principles is known as the APT (Arbitrage Pricing Theory).

An investment is profitable if it equals or surpasses the discounted value of the cash-flows it is expected to generate in the future. Finance theory requires that the discounting of future expected cash-flows ought to be carried out using discount rates similar to those observed in assets bearing the same risk. If there is a model relating risk to expected return, and if the way assets are similar before risk is known, the discounting of future cash-flows can be estimated, thus allowing thorough investment decisions. Otherwise, it becomes an empirical exercise and probably leads to wrong decisions.

When an asset is traded in capital markets, the former requirement is fulfilled with the model outlined above, based on the impossibility of sustained arbitrage, or using other models. But the relation that the market establishes between sensitivities to unanticipated changes in forces impinging upon it and the different possible characteristics of every asset, is not well understood yet. Even restricting ourselves to spe- 
cific groups of assets like industries, there is no available guidance to relate expected returns to the features of assets. As a consequence, it is impossible to find out what makes assets similar before risk.

This study tries to fill this gap. It shows that it is possible to build a taxonomy of risk. There is a strong, though non-linear, relationship between assets's characteristics as perceived by the market and the way they are traded.

\subsection{Non-Diversifiable Risk}

Assets are traded by what they are worth in the market's eyes. But the holders of traded assets are not risking their wealth in a way that directly relates to the risk of each asset they hold. Just by holding many assets, investors smooth out a great deal of the variability of their wealth. The only variability they can't avoid is the background movement of the whole market.

This background movement is a unique time-history. But it can be viewed as the result of a few orthogonal forces or "factors", each one of them capturing the largest dimension of the joint variability of returns on all the traded assets. These factors are obtainable from a representative number of time-histories of returns, by decomposing their joint variability into main orthogonal co-movements. Each factor is supposed to be a linear combination of economic movements like Inflation, Industrial Production, Risk Premium in the market, and Interest Rate's term structure growth.

After Ross's seminal paper in 1976 [10], the models using arbitrage considerations to relate risk to return (the APT), became present in the finance literature but scarcely in the finance practice. The first signs of their practical use only nowadays seem to emerge. The reason for this delay may well be the immediate interest of early models, based on one-dimensional sensitivities to non-diversifiable risk (the Betas). It is clear that, if a thoroughly calculated Beta really expresses the co-variance of an asset's return with the whole movement of the market, then investors don't need anything else. The co-variance with the market is the only portion of an asset's variability that investors will pay to avoid. Thus, it is also the only piece of information they are likely to pay to know.

However, there are cases in which multi-dimensional models based on arbitrage could become useful for financial practice. Whenever a more precise understanding of the way assets are traded is required, the APT is the choice. This is the case for the appraisal of productive commitments. It is also the case of studies concerned with the market itself.
When searching for a taxonomy of risk, arbitrage models seem promising. They are a breakdown of an information content into features. They should allow a better discrimination, each of the orthogonal forces showing particular affinity towards some aspects of assets. The APT offers the possibility of looking onto an asset's expected return from several points of view. When the emphasis is not in the holder but in the asset itself, the APT is the adequate instrument for assessing the variability of returns. That's why it's used here.

\subsection{Stable and Fluct uating Features}

The hypothesis that the market regards differently the stable features of assets and the more fluctuating ones, is a starting-point in our search for a taxonomy of risk.

A rapidly growing body of research documents components which can be forecasted in asset's returns (see for example Fama (1988) [4]). It is reasonable to divide investors's movements in two categories: Their expectations, creating the predictable component of returns, and their reaction to unanticipated events, which creates the unpredictable one. Arbitrage considerations (the APT) lead to models in which sensitivities to unanticipated economic forces play a role: Risk is about the second component of returns.

The expected component of returns mainly relates to changes in accounting numbers. For example, an increase or decrease in Earnings or Dividends influence expectations (see, for example, Brown and Ball, 1967 [2]). However, the features of the firm aren't equally volatile: There are pieces of accounting information that cannot vary swiftly as they reflect features of the firm that are stable.

We think that the firm's stable attributes are the ones that mainly create in investors the sensitivity to unanticipated forces. In fact, only stable features can create sensitivity: The fluctuating ones aren't sensitive as they move along with the trends. For example, the industrial group would explain to some extent the sensitivity of investors to unexpected movements of the economy, because firms cannot easily change sector to face inflation or an excess of supply. Conversely, less intrinsic features, like the financial structure or the dividend policy, relate to trends and thus to expectations observed in returns.

Hence, this study should search for relations between the stable features of assets and their sensitivities to market forces. We consider industry group, location, size, operating leverage, labour and capital intensity, payment pattern (hence, the short-term debt) 
as candidates for explaining market sensitivity. Dividend policy, capital structure, are less intrinsic. Earnings could have both components.

\subsection{Existing Research}

This study isn't a replica of an existing one, in which an MLP is used instead of linear modelling tools. It gives a new formulation to an old problem. Such a formulation is partly possible because of the MLP. It mainly concerns three areas of research: The searching of correlations between characteristics of the firm and risk, the building of proxies for Beta based on acounting information, and the APT. Foster (1986) [5] review them.

An early attempt to build a proxy for Beta using accounting numbers is the one of Beaver, Kettler and Scholes (1970) [1]. They achieved small but significant correlations between accounting and market measures of risk. Much research has also been published on the correlation of operating leverage, gearing, size, with Beta, the covariance of an asset's expected return with the background movement of the whole market. $30 \%$ is a typical value for the proportion of variability of returns explained by these models.

We would like to underline the fact that models like those of "Fundamental Analysis" (which assumes that each asset has an intrinsic value that can be determined on the basis of earnings, dividends, capital structure and potential growth) or "Equity Valuation" (which focus on predicted earnings and dividends), are not the kind of relation we are interested in here. Such models calculate indexes reflecting trends observed in individual histories of firms and use them to explain the market reaction to the performance of those firms. Since we focus on risk, that is, the unanticipated components of returns, we are not trying to forecast returns or other indexes.

\section{Preliminary Processing of Data}

This study brings together two sources of data. Firstly, a large set of time-histories of returns on assets traded in the London Stock Exchange, from which sensitivities of a few of them to four orthogonal forces are extracted. Secondly, the collection of accounting reports of the assets whose sensitivities were extracted. The sensitivities are the ones we use in the next section as target or explained variables, both in the MLP and in linear regressions. The accounting data will be used as input.

\subsection{Time-Histories of Returns}

The assets selected for extracting sensitivities were listed in the FTA All Shares Index. The reason for using only listed firms relates to the decision of avoiding infrequently traded assets. The known means for circumventing the problems posed by infrequent trading [3] would introduce in our experiment an extra manipulation of information. For the same reason, the use of daily or weekly returns was discarded. We used monthly returns provided by the London Business School, spanning a period of 15 years, from January 1974 to December 1988. Returns were calculated as

$$
r_{t}=\log _{e}\left(\frac{p_{t}+d_{t}}{p_{t-1}}\right)
$$

in which $p_{t}$ stands for traded price in month $t, p_{t-1}$ is the last traded price in month $t-1$ and $d_{t}$ is the dividend declared adjusted to a month-end basis. All adjustments were based on the principle that the value of a share is unaltered by a change in capital structure.

We used a total of 121 time-histories of assets for the extraction of sensitivities. All of them were industries. Included in these 121 firms were the 77 ones whose accounting reports were to be related to their sensitivities. The selection of the 77 final firms was made on an industry basis. Only seven industries were selected. Since one of our goals was the assessment of the effect of industrial grouping, it didn't seem appropriate to gather in the same sample a large number of industries. The selected ones were:

\begin{tabular}{|l|c|l|c|}
\hline Industry & N. firms & Industry & N. firms \\
\hline Building Materials & 11 & Electronics & 9 \\
Paper and Packing & 10 & Textiles & 10 \\
Chemicals & 15 & Food Manuf. & 13 \\
Electrical & 9 & & \\
\hline
\end{tabular}

All companies quoted on the London Stock Exchange are classified into industrial groups according to the Stock Exchange Industrial Classification (SEIC). The above groups refer to the SEIC.

Given the returns of 121 industries during the referred period, a Factor Analysis was performed on their time-history taken as stochastic variables. The aim was to rotate the co-variance matrix so that a few minimum co-variance axis would emerge. Thus, we obtained a breakdown of the cross-correlation of time-histories into its four main components. In order to maintain comparability with other APT studies we used Maximum-Likelihood as the criterion for the extraction of factors.

The resulting factors are supposed to be a linear combination of a few economic forces impinging upon 
the market. In our case the four main factors explained $47 \%$ of the variability of returns. Their Eigenvalues (in percent of the explained variability) are:

\begin{tabular}{|c|r|c|}
\hline factor & Eigenvalue \% & Accumulated $\%$ \\
\hline 1 & $39.5 \%$ & $39.5 \%$ \\
2 & $3.0 \%$ & $42.5 \%$ \\
3 & $2.4 \%$ & $44.9 \%$ \\
4 & $2.0 \%$ & $46.9 \%$ \\
\hline
\end{tabular}

The communality of firms ranges from $15 \%$ to $75 \%$. It is approximately Gaussian with a mean of $50 \%$ and a standard deviation of $11 \%$. That is, in these industries half the variability of their returns is captured by the four selected factors.

The factor loadings, not the factors themselves, are the main object of this experiment. They are supposed to represent the sensitivity of assets to unanticipated changes in market forces: Large loadings mean a sensitive asset. When a loading is negative, the influence and the force go in opposite directions. Any anticipated return is incorporated into the expected value of returns and it is accounted for before the extraction of factors.

We now describe the statistical behaviour of each sensitivity when considering solely the 77 selected firms. The four variables containing the sensitivities of firms are homogeneous. No influential firms were found. Next table shows their statistics.

\begin{tabular}{|c|c|c|c|c|}
\hline $\begin{array}{c}\text { Sensitiv. to } \\
\text { Force N. }\end{array}$ & Skewness & Kurtosis & Mean & St. Dev. \\
\hline 1 & -0.88 & -0.12 & 0.412 & 0.189 \\
2 & -0.48 & 0.19 & 0.269 & 0.124 \\
3 & 0.40 & 0.60 & 0.263 & 0.148 \\
4 & 1.88 & 1.03 & 0.184 & 0.126 \\
\hline
\end{tabular}

There are no strong differences between the sensitivities in what concerns these values. The fourth sensitivity is less homogeneous than the desirable.

Sensitivities are not correlated with each other. Despite the factors being orthogonal, the sensitivities they generate could be correlated, but they aren't. Only the fourth sensitivity shows non-significant traces of negative correlation with the first and second ones.

The effect of industrial grouping is very significant in the first three sensitivities, and it is weak in the fourth. The intra-class correlations (see Snedecor and Cochran 1965, [13] $9^{\text {th }}$ edition, page 242) are:

\begin{tabular}{|c|c|c|c|}
\hline Factor 1 & Factor 2 & Factor 3 & Factor 4 \\
$35.4 \%$ & $39.9 \%$ & $43.1 \%$ & $3.4 \%$ \\
\hline
\end{tabular}

Using the convention of calling "positive" to influences which are above the expected and "negative" otherwise, we can say that Factor 1 strongly influences

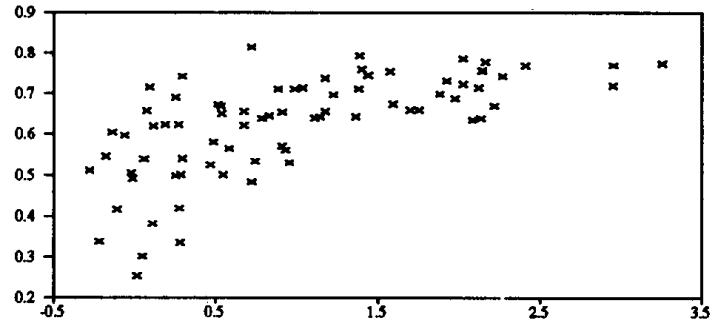

Figure 1: The first factor's sensitivities (Y-axis) against $\sigma_{j}$, the relative size of firms (X-axis).

prices on the Paper industry. It is also very influential for the Food industry, but in the opposite direction. Factor 2 has a positive influence in Food Manufacturers and Electronics. It has a negative or no effect on the other industries. Factor 3 has a strong, negative, influence on the Electronics industry. It is a positive one for Food Manufacturers and Paper.

We also observed the correlations between the communality associated with each firm, their loadings and some features of these firms. The communality measures the portion of variability of returns that are explained by the factors. In our sample, it clearly relates to Size and Earnings. This means that the largest firms and the most profitable ones are also the ones traded in a more similar, predictable, way. An interesting study related to this issue is Roll's " $R^{2}$ " (1988) [8]. It is an empirical assessment of the variability explained in stock returns by the market models. Roll correlates the collection of $R^{2}$ observed in several kinds of market models with a few characteristics of the firm.

Scatter-plots of features of firms against their sensitivities were also observed as part of this preliminary study. Correlations were observed between Size and the first two factor's sensitivities. The correlation between Size and the first one is non-linear (see figure 1 on page 4). The second one exhibits a negative, linear, correlation with size but industrial groups form visible clusters. Also Gross Funds From Operations and Inventory show significant correlation with some sensitivities.

\subsection{Accounting Reports}

Accounting raw data, as found in databases containing collections of annual reports of firms, can be viewed as statistical variables. Each firm is a case. For a given item, say, Fixed Assets or Sales, a collection of reports from the same year forms a cross-sectional sample. The probability density function governing 
such variables is the lognormal one. Items belonging to the same report share, in cross-section, most of their variability. This is caused by the prevalent effect of size. Size is an important piece of information but its effect isn't directly measurable. However, it is possible to isolate it by building, inside each report, averages of a few positive-valued items like Net Worth, Wages or Sales. In this way, we self-smooth the particular scatter of each item so that only the common one remains.

Thus, considering a group of accounting items, $x_{j}^{i}, i=1, M$, from the $j^{\text {th }}$ report, we explain their variability as the result of an effect, $\sigma_{j}$, common to them all, plus the residuals, $\varepsilon_{j}^{i}$, particular to each item:

$$
\begin{array}{ccc}
\log x_{j}^{1}-\mu_{1} & = & \sigma_{j}+\varepsilon_{j}^{1} \\
\log x_{j}^{2}-\mu_{2} & = & \sigma_{j}+\varepsilon_{j}^{2} \\
\vdots & & \vdots \\
\log x_{j}^{M}-\mu_{M} & = & \sigma_{j}+\varepsilon_{j}^{M}
\end{array}
$$

The $\mu_{i}$ are the expected values of $\log x^{i}$. The average of the $1, \cdots, i, \cdots, M$ mean-adjusted items case by case yields, for report $j$,

$$
\sigma_{j}=\frac{1}{M} \sum_{i=1}^{M}\left(\log x_{j}^{i}-\mu_{i}\right)-\frac{1}{M}\left(\varepsilon_{j}^{1}+\varepsilon_{j}^{2}+\cdots+\varepsilon_{j}^{M}\right)
$$

Since an average of independent random deviates tends to zero with $1 / M$,

$$
\sigma_{j} \approx \frac{1}{M} \sum_{i=1}^{M}\left(\log x_{j}^{i}-\mu_{i}\right)
$$

for large enough $M$. Once obtained, $\sigma$ allows the assessment of the $\varepsilon^{i}$ particular to each item:

$$
\varepsilon_{j}^{i}=\log x_{j}^{i}-\mu_{i}-\sigma_{j}
$$

In this study, the estimated $\sigma$ was obtained as

$$
\begin{aligned}
\sigma= & \frac{1}{7}[\log S+\log N W+\log W+\log N \\
& +\log D+\log C A+\log C L]
\end{aligned}
$$

The meaning of the abbreviations can be found in table 1 . The log items were mean-adjusted separately by industry and by year before being used in formula (2). Thus, the industry and year effects aren't present in these variables. A more detailed characterisation of the statistical behaviour of accounting data can be found in Trigueiros (1992) [15].

We used the $\varepsilon^{i}$, along with our proxy for size, the $\sigma_{j}$, as input variables to explain sensitivities. Notice that the $\varepsilon^{i}$ are the logarithm of ratios in which the

\begin{tabular}{|ll|ll|}
\hline FL & Gr. Funds fr. Operat. & NW & Net Worth \\
FA & Fixed Assets & DB & Long Term Debt \\
D & Debtors & C & Creditors \\
CA & Current Assets & CL & Current Liabil. \\
I & Inventory & S & Sales \\
N & Number of Empl. & W & Wages \\
\hline
\end{tabular}

Table 1: List of accounting items used in this study and their abbreviations.

denominator is the statistical effect of size and the numerator is the deviation of item $i$ from the standard in its industry. Such ratios reflect the proportion to which the item in the numerator departures from the expected for firms of that size. That is, the $\varepsilon^{i}$ are size-adjusted Sales, Working Capital and so on. Apart from rendering Gaussian inputs, the use of log space makes the $\varepsilon^{i}$ similar to proportions: The deviations from size they assess are independent of scale and unit of measure.

\section{The Method}

We modelled sensitivities in terms of accounting information, firstly using linear regressions and then the MLP. In both cases the training sets were the same. Since the four targets are almost orthogonal there is nothing to be gained from showing them jointly to the modelling tool. Hence, we built four independent MLPs, each one for explaining one sensitivity.

\subsection{Input Variables}

Table 2 on page 7 shows the 17 input variables used both in the linear regressions and in the MLP. Nine of them are the $\varepsilon^{i}$, obtained from applying formula (1) to accounting items. One is our proxy for size, the $\sigma_{j}$, as calculated by formula (2). The remaining seven are dummy variables taking the value 1 when a case belongs to a particular industry and 0 otherwise.

The selection of the set of nine items was a result of an exploratory study, similar to the one presented here but involving a larger number of items and different topologies. The penalization of small weights, to be described in section 3.2 , allowed the elimination of non-significant input variables.

The accounting data were drawn from the MicroEXSTAT database of company financial information provided by EXTEL Statistical Services Ltd, which covers the top $70 \%$ of UK industrial companies. As mentioned above, we used the reports of 77 firms matching the selected sensitivities. For each firm, four years of accounting reports were collected: 1983 


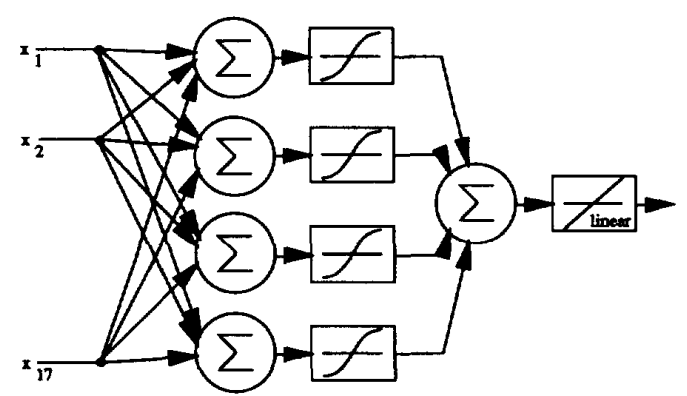

Figure 2: Schematic representation of the used MLP.

to 1986. Each example in the training set contains the accounts of one firm for one of these years and having as target one sensitivity. Therefore, there are $77 \times 4=308$ examples.

\subsection{The MLP}

In many MLP applications the problem is one of classification. In this case it was one of regression. The only difference, in what concerns the MLP, is that linear transfer functions must be used in the output layer of nodes and that the adequate success criterion is Least-Squares minimization, not Likelihood maximization.

The topology of the MLP and the input variables were similar in the four cases (see a schematic representation in figure 2). There were 17 input nodes, corresponding to 17 input variables. The unique hidden layer had four nodes and the output layer had a linear transfer function. This topology seems austere. But since the number of cases in the training set was small, the number of parameters in the models had to be small as well. The characteristics which make our MLP different from the standard algorithm (Rumelhart et alt. 1986 [11]) can be summarized as:

- The use of two samples, one to learn and another one to assess the classification performance, along with incomplete training.

- Penalization of small weights, as described by Trigueiros and Berry (1991) [16].

- Learning rates particular to each weight as described in Silva and Almeida (1990) [12].

We now comment on these characteristics. The first one relates to improvements in the ability to generalise. It is a particular implementation of a known procedure, the Cross-Validation (see Stone 1978 [14]). In order to obtain an estimate of the generalisation capacity of a model, the original samples were divided randomly into two sub-samples. Models were constructed with one sub-sample, the training set, and a check carried out with the other one, the test set. Since the outcomes were continuous-valued, the adequate measure of quality of fitting was the proportion of explained variability, $R^{2}$, corrected for the degrees of freedom engaged. All reported results concern the test set, not the training set. That is, they were obtained by measuring the rate of explained variability in the half-set not used for training. The performance on the set used for training depends solely on the number of free parameters and can be increased simply by introducing more nodes on the net. Therefore such results are uninteresting.

The randomization leading to the division into two samples - the test and the training set - was carried out by firms on an industry basis. Thus, the two resulting groups of firms didn't yield large differences in their proportion of cases for each industry. The training set contained $2 / 3$ of the firms.

Since the MLP seeks an optimum iteratively, we can stop its training when an optimum is obtained in the test set rather than in the training set. We thus prevent this powerful algorithm from over-fitting the data. The Back-Propagation algorithm seeks the modelling of progressively smaller or less important features of the relation, during the learning process. Firstly, broad features are accounted for: The mean, a linear trend. Then, more detailed ones are modelled. Hence, the effective degrees of freedom the MLP engages can be viewed as increasing during learning. Assuming that the topology of the net contains plenty of free parameters, the MLP will be able to model, not only the desired features but also the undesirable random uniqueness of a particular sample. We prevent it from doing this by stopping the process before finishing. The appropriate moment for stopping is when the results, as measured by the test set, are optimal. For a good topology, the fact that the learning stops before a minimum is reached in the learning set clearly enhances generalisation.

Finally, we introduced during training a penalization of weights smaller than 1 in order to enhance interpretability. If the input variables were very differently scaled, weights smaller than 1 could just mean that the MLP was trying to scale down a particular variable. Since the input to the MLP are meanadjusted and have similar standard deviations, the only reason for any such weights to remain small throughout the learning is to try to diminish the importance of one variable to the output of the node it 


\begin{tabular}{|c|c|c|c|c|c|c|}
\hline \multirow[t]{2}{*}{ Input variable } & & \multicolumn{4}{|c|}{ Number of parameters engaged } & \multirow[t]{2}{*}{ Total } \\
\hline & & Model 1 & Model 2 & Model 3 & Model 4 & \\
\hline Chemical & (dummy) & 3 & 1 & 1 & 1 & 6 \\
\hline Electronics & (dummy) & 2 & 3 & 1 & & 6 \\
\hline Size & $\left(\sigma_{j}\right)$ & 2 & 2 & & 2 & 6 \\
\hline Textiles & (dummy) & 2 & 2 & & 2 & 6 \\
\hline Wages & $\left(e^{i}\right)$ & 3 & 1 & 2 & & 6 \\
\hline Current Assets & $\left(e^{i}\right)$ & 3 & 1 & 1 & & 5 \\
\hline Debtors & $\left(e^{i}\right)$ & 2 & 1 & & 2 & 5 \\
\hline Electrical & (dummy) & 1 & 1 & 2 & 1 & 5 \\
\hline Net Worth & $\left(e^{i}\right)$ & & 3 & 1 & 1 & 5 \\
\hline Paper and Packing & (dummy) & 3 & 1 & & 1 & 5 \\
\hline Building Materials & (dummy) & 3 & & & 1 & 4 \\
\hline Sales & $\left(e^{i}\right)$ & 1 & 2 & & & 3 \\
\hline Fixed Assets & $\left(\varepsilon^{i}\right)$ & 1 & & 1 & & 2 \\
\hline Food Manufacturers & (dummy) & 1 & 1 & & & 2 \\
\hline Gross Funds From Ops. & $\left(e^{i}\right)$ & 1 & 1 & & & 2 \\
\hline Debt & $\left(\varepsilon^{i}\right)^{\prime}$ & 1 & & & & 1 \\
\hline Inventory & $\left(\varepsilon^{i}\right)$ & & & & 1 & 1 \\
\hline
\end{tabular}

Table 2: The number of parameters (first hidden layer) engaged during the training of the four MLP, by input.

belongs to. In the MLP, each node acts as a modelling unit with a certain amount of free parameters. The same output can be obtained with very different combinations of weights. Small weights connecting inputs with the first hidden layer appear when a node tries to weaken the contribution of a variable. If we introduce a penalization of such weights during the training, as the correction of weights is proportional to the input variables, small weights tend to remain small. In the same way, large weights tend to have their values strengthen. The final result is a contrasted set of weights: The first layer now contains only very large or very small weights. The information concerning the modelled relation is concentrated in a few weights instead of distributed by all of them [16].

Notice that we never tried this method with the simple Back-Propagation. Each one of the weights in our MLP has its own increment, adjusted as described in Silva and Almeida (1990) [12]. Other popular methods for pruning the MLP are the "Skeletonization" [7] and "Optimal Brain Damage" [6]. However, the first one is intended to reduce the number of nodes, not the one of weights. The second one is too general for this task.

\section{The MLP Versus Linear Regression}

Table 2 shows, for each one of the four models, the number of non-zero weights in the first hidden layer, when the training finished. The remaining ones were set to zero by the algorithm described above and play no role in the modelling. The non-zero weights are the parameters of the first hidden layer engaged during the training of the MLP. Since the number of nodes in the first hidden layer was four, the maximum number of parameters per input variable that the MLP could engage in the modelling process would be four as well. This never happened. The training started with 68 free parameters and finished with 29 remaining ones (the first model), 20 (the second), 9 (the third), and 12 (the fourth).

The number of parameters engaged is a rough measure of the complexity of the relation. Size and Wages engage a total of six parameters. Debt and Inventory merely engage one. In general, the dummy variables engage more parameters than items. Next table compares the proportion of variability, $R^{2}$, explained by all models, when using linear regressions (OLS) and the MLP.

\begin{tabular}{|l|c|c|c|c|}
\hline$R^{2}$ & Model 1 & Model 2 & Model 3 & Model 4 \\
\hline MLP & $\mathbf{7 1 \%}$ & $\mathbf{4 8} \%$ & $69 \%$ & $36 \%$ \\
OLS & $60 \%$ & $42 \%$ & $52 \%$ & $21 \%$ \\
\hline
\end{tabular}

The comparing of the $R^{2}$ achieved by the MLP with those of linear regressions lead us to conclude that the MLP made a significant improvement in capturing the relation. In next section we shall see why: Such a relation contains higher order effects and other sources of complexity.

\section{Exploring the Models}

This section describes how the exploring of the four models was carried out. We used an "other things being equal" approach. It consisted of varying one of the inputs at a time, and maintaining all the others fixed 

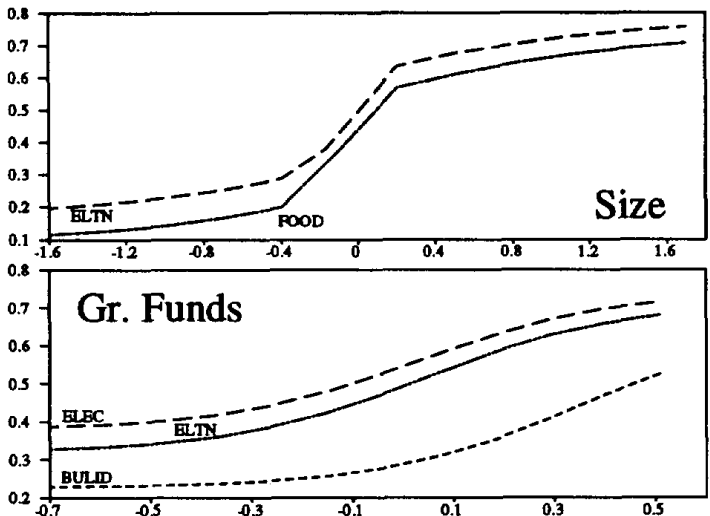

Figure 3: The first factor's sensitivity to Size (above) and to Gross Funds From Operations (below) as predicted by the MLP.

in their mean values. The observed outputs could then be compared.

The chosen approach is the only one available in this case. The model the MLP yields is far too complicated for direct interpretation. The used procedure allows an effective look into what the model does. However, in some cases, the "other things being equal" technique yields misleading results since the other things cannot remain equal in the real world.

We firstly comment on the simplest relations - the ones which are linear regarding industrial grouping. Then, we comment on those cases in which the model shows industries reacting in different directions to the same force.

Figure 3 shows, for a few industries, the relation modelled by the MLP when explaining the first factor's sensitivity to Size (above) and Gross Funds From Operations (below). Similar patterns were obtained for Sales and Long Term Debt (a negative correlation). Each line represents an industrial group. Notice that, since we used size-adjusted items as input variables (the $\epsilon^{i}$, formula 1 ), a value of zero means the expected for firms of that size. Values larger than zero mean Sales or Gross Funds above the expected.

Electronics is the industry whose size has the largest sensitivity to the first economic force, while Building Materials is the one with the smallest. However, since in Factor Analysis the sign of the factors is arbitrary, the displayed curves yield a coherent view but not one directly comparable with studies based on correlations with Beta. For example, the trends we observe in the case of the first factor are the inverse of the ones Beaver, Ketler and Scholes (1970) [1]
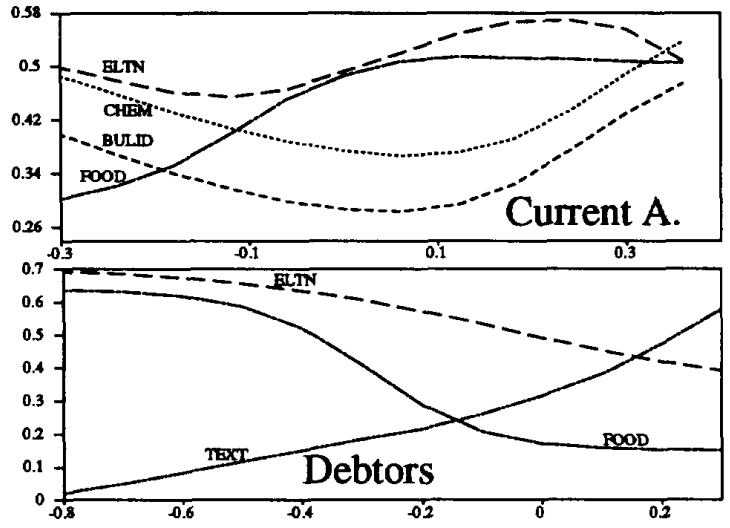

Figure 4: The first factor's sensitivity to size-adjusted Current Assets (above) and Debtors (below) as predicted by the MLP.

obtained for similar variables.

The first factor rewards positive features of the firm and penalizes Debt. None of the displayed curves deserved from the MLP the engagement of many parameters as these are easy-to-build relations. The second factor rewards Wages, Debtors and penalizes Earnings. But some industries are more sensitive than others. Again, remember that the meaning of Wages or Sales being large is that they are large given the size of the firm. The third factor's sensitivities reward Net Worth and penalize Fixed Assets. Finally, the fourth factor's sensitivities penalize Size and Debtors and reward Inventory but in a non-linear way.

So far, we described the cases in which the sensitivities of all industrial groups react in the same direction to the same orthogonal force. If we examine the number of parameters engaged by the MLP we notice that, except for Size, none of the relations commented above deserved the use of many degrees of freedom. Size needed more degrees of freedom as it is a strong non-linear though monotonic relation. But it is similar across industries.

We now study a few cases in which the MLP put a great effort. The first one is displayed in figure 4 on page 8 . It is clear that the first factor's sensitivities reward Debtors for industries like Textiles or Paper and penalize it for industries like Food Manufacturers. The interaction of Current Assets with the first force is particularly awkward. It seems as if there is a penalization of the values expected for a given size in industries like Chemicals and Building Materials along with a rewarding of the same situation in Electronics and Food. 
Factor 2 also displays this higher-order behaviour. Figure 5 on page 9 shows the complicated pattern of its relation with Net Worth and Size. Again, industries seem to be affected in different directions by the same market force. It rewards Net Worth if it is the expected one for firms of that size, in industries like Textiles and Paper. But it penalizes the same situation if the industry is Food. In the Electronics industry this force simply rewards capital. A standard size is rewarded by this factor if the industry is Textiles. It is penalized if it is Food. Small firms are, in general, rewarded by this factor. But there is one exception, Textiles. On the contrary, in the case of Electronics firms, the smaller the better - with regard to the second factor.

The third factor's sensitivities reward Wages if near the expected, penalizing both higher and smaller ones. Fixed Assets of the Electronics industry seem not to be affected by this force.

\section{Towards a Taxonomy of Risk: Dis- cussion and Conclusions}

The sensitivities of assets to unanticipated market forces seem to be closely related to features identifiable and measurable in assets. Size and industrial grouping are the characteristics of assets which mostly explain sensitivities. Deviations from the expected for size in Wages, Net Worth and Sales, along with the short-term structure, also relate significantly to sensitivities. However, the relation between sensitivities and features is complex. The industrial grouping interacts with features like Net Worth, Current Assets or size. The same market force reacts in opposite directions to the same feature in different industries.

To some extent, particular forces impinge upon specific features. The fourth orthogonal force is the only one relating to Inventory. The third one relates mainly to Wages and the second one to Net Worth. The first and second forces's sensitivities react to Size in opposite directions. All of them recognize industrial grouping but the fourth one to a small extent.

The ability of the MLP to model higher order relations achieved a significant improvement in our knowledge of the way the market recognizes and rewards assets. However, the results were obtained from less cases than desirable and further experiments involving more firms should be carried out.

The fact that our sample is a dated one - it belongs to a period of stability and expansion - seems fortunate. The modelling of these relations would be diff-

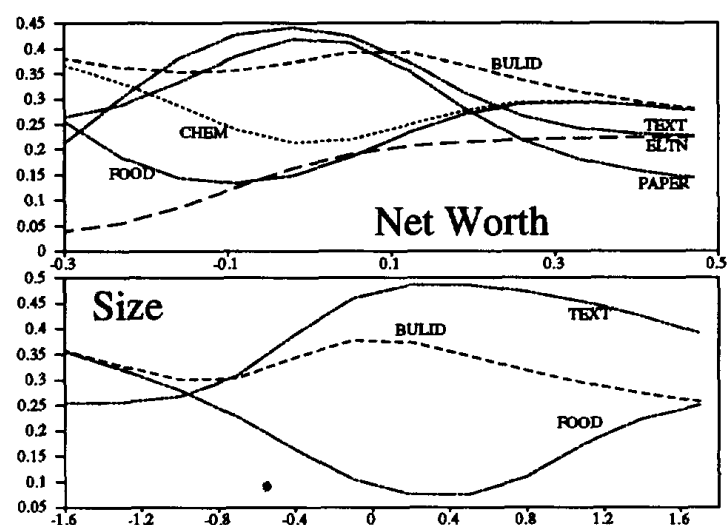

Figure 5: The second factor's sensitivity to sizeadjusted Net Worth (above) and Size (below) as predicted by the MLP.

cult to carry out in data from recessive periods as the number of non-traded assets would greatly increase. The firms used are also from a particular set. They are large and frequently traded. They were drawn from well known and homogeneous industries. Our results refer to such set and shouldn't be extrapolated. The behaviour of returns on less important assets is an in teresting issue but there isn't enough information to clarify it using statistical methods.

There are other studies of this kind in the accounting and finance literature. Though they found significant correlations between accounting and market risk variables, their proportions of explained variability were smaller than ours. The reasons for this are several: Instead of using ratios, we defined an input space based on a statisticaly sound analysis. It may well be that such an input is suited for modelling to an extent so far unattained by other studies. Also, we modelled co-variability, not indexes extracted from individual histories. Thus, we avoided using pieces of information the market regards as predictable. Most of the existing studies use earnings, dividends and their standard deviations to predict Betas. But these variables are, to some extent, predictable. They relate to the policy and quality of management. They have little in common with unanticipated components of overall forces. We have shown that the way investors react to unanticipated changes mainly depends on stable characteristics of assets. Finally, we modelled four different points of view, not just one, thus obtaining a better scanning of the variability of returns, and we used non-linear tools for modelling relations that are complex.

It seems now clear that there is room in finance re- 
search for the use of MLPs. They apportion significant improvements in the amount of explained variability in crucial problems as they model higher order relations without damaging generalisation.

Our results also show that it is possible to build, for each group of assets, a taxonomy of risk in terms of their characteristics. Expected returns can then be calculated on the basis of such characteristics allowing a less blind discounting of future cash-flows. For example, if a productive commitment is to be undertaken, the models produced by the MLP could be fed with its budgeted numbers. As a result they would yield four sensitivities. Then the APT would predict its return based on these sensitivities.

Finally, it seems also possible to achieve a better understanding of the forces impinging upon the market by examining the features of firms and industries affected by each force.

Acknowledgements: We acknowledge the guidance and continued support of Professor Luis B. Almeida at INESC, Lisbon. The experiments described in this study and some developments that followed, were carried out in the University of East Anglia in the UK. We thank Professor Robert H. Berry for his valuable support.

\section{References}

[1] W. Beaver, H. Kettler, and M. Sholes. The association between market-determined and accounting-determined risk measures. The $A c$ counting Review, pages 654-682, October 1970.

[2] P. Brown and R. Ball. Some Perliminary Findings on the Association Between the Earnings of a Firm, its Industry, and the Economy. Empirical Research in Accounting: Selected Studies, 1967. Suplement to Journal of Accounting Research.

[3] E. Dimson. Risk measurement when shares are subject to infrequent trading. Journal of Financial Economics, 7:197-226, 1979.

[4] E. Fama and R. French. Dividend yields and expected stock returns. Journal of Financial Economics, 22:3-25, 1988.

[5] G. Foster. Financial Statement Analysis. Prentice-Hall, 1986.

[6] Y. LeCun. Optimal brain damage. In Neural Information Processing Systems, volume 1. Morgan Kaufmann, Denver 1990.
[7] M. Mozer and P. Smolensky. Skeletonization: A technique for trimming the fat from a network via relevance assessment. In Neural Information Processing Systems, volume 1. Morgan Kaufmann, Denver 1988.

[8] R. Roll. $R^{2}$. The Journal of Finance, XLIII(2):541-567, July 1988.

[9] R. Roll and S. Ross. The arbitrage pricing theory approach to stratergic portfolio planning. Financial Analysts Journal, pages 14-26, May 1984.

[10] S. Ross. The arbitrage theory of capital asset pricing. Journal of Economic Theory, 13:341-360, 1976.

[11] D. Rumelhart, G. Hinton, and R. Williams. Learning internal representations by error propagation. In Parallel Distributed Processing, volume 1. The MIT Press, 1986.

[12] F. Silva and L. Almeida. Acceleration techniques for the backpropagation algorithm. In Neural Networks. EURASIP Workshop, Sesimbra, Portugal, 1990. L. B. Almeida and C. J. Wellekens (Eds.), Springer-Verlag.

[13] G. Snedecor and W. Cochran. Statistical Methods. Iowa State University Press, $9^{\text {th }}$ edition, 1965.

[14] M. Stone. Cross-validation: A review. Math. Operationsforsch. Statist., Ser. Statistics, 9(1), 1978.

[15] D. Trigueiros. The Cross-Sectional Characterization of Accounting Data. Technical Report, ISCTE, Lisbon, Portugal, 1992.

[16] D. Trigueiros and R. Berry. The application of neural network based methods to the extraction of knowledge from accounting reports. In E. Nunamaker and R. Sprague, editors, Organisational Systems and Technology: Proceedings of the $24^{\text {th }}$ Hawaii International Conference on System Sciences, pages 136-146. IEEE Computer Society Press, 1991. 